\title{
La socialisation entre groupes professionnels de la politique étrangère
}

Le cas de l'institutionnalisation des activités civilo-militaires françaises en ex-Yougoslavie

The socialization between the professional groups of the foreign policy. The case of the institutionalization of the French civil-military activities in former

Yugoslavia

\section{Grégory Daho}

\section{(2) OpenEdition}

\section{Journals}

Édition électronique

URL : http://journals.openedition.org/conflits/19018

DOI : 10.4000/conflits. 19018

ISSN : $1777-5345$

Éditeur :

CECLS - Centre d'études sur les conflits - Liberté et sécurité, L'Harmattan

Édition imprimée

Date de publication : 15 octobre 2015

Pagination : 101-131

ISBN : 978-2-343-07829-8

ISSN : 1157-996X

Référence électronique

Grégory Daho, "La socialisation entre groupes professionnels de la politique étrangère ", Cultures \& Conflits [En ligne], 98 | été 2015, mis en ligne le 15 octobre 2016, consulté le 02 avril 2021. URL : http:// journals.openedition.org/conflits/19018; DOI : https://doi.org/10.4000/conflits.19018 


\title{
La socialisation entre groupes professionnels de la politique étrangère
}

\author{
Le cas de I'institutionnalisation des activités civilo-militaires \\ françaises en ex-Yougoslavie
}

\section{Grégory DAHO}

Grégory Dabo est Docteur en Science Politique de l'université Paris 1 et membre du Centre Européen de Sociologie et Science Politique (CESSP Paris). Ses travaux portent sur la sécurité internationale, la transformation des organisations et des doctrines militaires et les interactions entre groupes professionnels de la politique étrangère et de défense. Il a récemment publié : L'érosion des tabous algériens. Une autre explication de la transformation des organisations militaires en France", Revue française de science politique, vol 64-1, février 2014.

et article porte sur la recomposition de la politique étrangère à travers
l'évolution des relations entre groupes professionnels spécialisés dans la
gestion des crises internationales. Qu'ils traitent des conséquences de l'élar-
gissement de la notion de sécurité ${ }^{1}$, de l'adaptation des dispositifs d'interven-
tion au caractère multidimensionnel des crises ${ }^{2}$ ou des effets de la conver-
gence des programmes internationaux sur les rapports de domination ${ }^{3}$, les
travaux axés sur les mutations des interventions concluent à une redistribution

1. Buzan B., People, States and Fear. An Agenda for International Security Studies in the PostCold War Era, New York, Hervester, 1991 ; Security: A New Framework for Analysis, Boulder, Lynne Rienner Publishers, 1998 ; Huysmans J., « Dire et écrire la sécurité : le dilemme normatif des études de sécurité ", Cultures \& Conflits, 31-32, 1998, pp. 177-202; Wæver O., "Societal Security: The Concept", in Wærver O., Buzan B., Kelstrup M., Lemaitre P. (eds), Identity Migration and the New Security Agenda in Europe, New York, St Martin's Press, 1993, pp. 17-40.

2. Ankersen C., Civil-Military Cooperation in Post-Conflict Operations: Emerging Theory and Practice, Londres, Routledge, 2008 ; Seybolt T., Humanitarian Military Intervention: The Conditions for Success and Failure, Oxford, Oxford University Press, 2007 ; Weiss T., Military-Civilian Interactions Intervening in Humanitarian Crisis, Lanham, Rowan \& Littlefield, 1999.

3. Ambrosetti D., "Autour d'un livre ", Politique africaine, 125, 2012 pp. 215-232 ; Chandler D., "Human Security II: Waiting for the Tail To Wag the Dog. A Rejoinder to Ambrosetti, Owen and Wibben”, Security Dialogue, 39/4, 2008, pp. 463-469 ; Duffield M., Development, Security and Unending War: Governing the World of Peoples, Cambridge, Polity Press, 2007. 
des rôles entre acteurs. Au regard de cette littérature, notre ambition est d'offrir une explication davantage incarnée, c'est-à-dire centrée sur les trajectoires, les positions et les interactions. Le fait que les relations entre militaires, diplomates et industriels aient beaucoup changé depuis la fin de la guerre froide peut sembler un constat banal. L'originalité de la démonstration consiste ici à ne pas déduire cette transformation de l'environnement post-bipolaire, mais plutôt de l'induire de l'observation de l'évolution des formes de socialisation, c'est-à-dire des processus d'intériorisation de normes et de références et d'assimilation entre groupes professionnels.

L'approche interactionniste permet de souligner l'homologie de trajectoires par delà les appartenances sectorielles. D'un côté, elle révèle non seulement l'existence de nombreux points communs entre un officier spécialisé dans le maintien de la paix, un diplomate chargé de promouvoir le développement et un industriel prospectant les marchés de la reconstruction mais aussi, et plus fondamentalement, un socle de valeurs, de représentations et de ressources partagées. De l'autre, elle met en évidence les compétitions entre groupes de fonctionnaires tout autant que d'improbables alliances bureaucratiques. Ces arrangements, fluctuant au gré des opportunités que constituent les interventions et les réformes, imprègnent de façon constante la division interministérielle du travail en matière de gestion des crises internationales. Notre objectif est de montrer les liens entre trajectoires, ressources et changement institutionnel dans un champ diplomatico-stratégique au final encore peu investi par ce type de démarche inductive et de souligner les dynamiques intersectorielles à l'œuvre dans l'autonomisation des groupes professionnels qui font la politique étrangère depuis la fin de la guerre froide.

Avec la mise en œuvre des politiques publiques liées à « l'approche globale ", les processus d'intégrations interministérielles et interservices entre la Défense, les Affaires étrangères et l'Économie se renforcent depuis la fin de la décennie 2000 dans la plupart des États et des organisations internationales $(\mathrm{OI})^{4}$. «L'approche globale » désigne un ensemble d'acteurs,

4. Grande-Bretagne (Leboeuf A., "Sécurité et développement : acteurs et consensus ", Afrique contemporaine, 218, 2006, pp. 69-83), États-Unis (Jasper S., Conflict and Cooperation in the Global Commons. A Comprehensive Approach for International Security, Washington, Georgetown University Press, 2012), France (Wendling C., L'approche globale dans la gestion civilo-militaire des crises, analyse critique et prospective du concept, Cahier de l'IRSEM, 2010) et Suède (Bjorkman T., Friman H., "Comprehensive Approach: The Swedish Case", in Neal D. (ed.), Capability Development in Support of Comprehensive Approaches. Transforming International Civil-Military Interactions, Institute for National Strategic Studies, National Defense University, December 2011, pp. 229-244). Les organisations internationales les plus actives sont l'ONU (De Coning C., "Implications of a Comprehensive or Integrated Approach for Training in United Nations and African Union Peace Operations" NUPI Working Paper 766, Security in Practice 6, 2009), l'OSCE (Novosseloff A., "Options for improving EU-UN cooperation in the field of peacekeeping", in Krause J., Ronzitti N. (eds), The EU, the UN and Multilateralism, 2012, Londres, Routledge), l'OTAN (Jacobsen P., "Right strategy, wrong place - why Nato's comprehensive approach will fail in Afghanistan?", UNISCI Discussion Papers, 22, 2010) et l'UE (Gourlay C., "Civilian crisis management: the EU way", Chaillot Paper, 90, 2006). 
d'organisations, de normes, de techniques mais aussi de critères de performance visant à améliorer les relations entre une multiplicité d'intervenants locaux, nationaux et internationaux, sur les zones d'opérations comme au sein des administrations centrales. Elle est conçue comme un outil permettant d'adapter les interventions extérieures à la complexité des crises internationales, dont la spécificité tiendrait précisément à l'hétérogénéité des catégories d'acteurs impliqués dans la fabrication des dispositifs d'intervention, de pacification et de transition politique. Les buts affichés sont l'anticipation et la gestion des crises internationales par une meilleure articulation entre ministères régaliens et entre acteurs publics et privés. Les administrations ministérielles n'ont jamais été hermétiques : au-delà de la sophistication des circuits de partage d'informations, la nouveauté de ces dernières années réside dans la fluidité de la circulation internationale des doctrines ${ }^{5}$, des modèles organisationnels ${ }^{6}$, des normes de gestion ${ }^{7}$ et des technologies administratives ${ }^{8}$. Les domaines au sein desquels ces fonctionnaires détachés par leurs ministères sont incités à «travailler ensemble » sont variés et en extension : politique gouvernementale, négociations internationales, coopération décentralisée, veille stratégique, placement d'experts dans les OI, collaboration avec les organisations non gouvernementales (ONG), programmes de financements, coordination avec les entreprises...

Quelque chose de fondamental se joue déjà vingt ans plus tôt en exYougoslavie pour cette cohorte de hauts fonctionnaires. L'implication française dans la gestion des crises en Bosnie et au Kosovo ne ressemble en rien aux expériences précédentes. Certes, ni les interventions multilatérales ni les activités militaro-humanitaires ne sont nées en ex-Yougoslavie et il convient de se méfier d'un double «effet laboratoire » : celui, pour les acteurs, d'une crise à « deux heures de vol de Paris », véritable opportunité de mise en situation pour une armée réformée, et celui pour le chercheur qui, reconstituant les évènements, aurait sans doute tendance à rationaliser l'unité et l'exemplarité de ces terrains. C'est cependant en Bosnie, entre 1992 et 1995, que les armées françaises vont faire l'expérience du maintien de la paix à grande échelle. La mission confiée sous mandat de l'ONU consiste à s'interposer entre les lignes ennemies dans une configuration de neutralité politique. Objet de nos recherches doctorales 9 , la coopération civilo-militaire (CIMIC), précisément expérimentée en Bosnie à partir de 1992, désigne l'ensemble des activités militaires tournées vers les acteurs civils lors d'une intervention, qu'ils soient

5. «Comprehensive ", «multidimensional ", « holistic ", «integrated» ou « global approach».

6. Agence, Task Force, comité de pilotage, mission interministérielle, cellule de crise, veille stratégique.

7. Accountability, relations usagers/clients, surveillance (watchdogs), évaluation par les pairs (peer review).

8. Avantages comparatifs, indicateurs de performance (GPEC, COP), RETEX, tableaux de bord, audits, expertises.

9. Daho G., Une revanche des généraux. L'institutionnalisation de la coopération civilo-militaire en France, thèse de doctorat de science politique, Université Paris 1 Panthéon-Sorbonne, 2013. 
locaux (belligérants, autorités, populations, entreprises, associations, administrations) ou allogènes (OI, ONG, agences de l'ONU, bailleurs de fonds, entreprises, services ministériels). Du point de vue des armées, la CIMIC est un outil qui permet de faciliter l'acceptation des forces militaires auprès des populations locales et l'accélération du transfert de responsabilités vers les autorités civiles ${ }^{10}$. L'observation des processus d'institutionnalisation de cette spécialité est instructive quant à l'évolution des relations entre acteurs de la politique étrangère.

L'analyse biographique par cohorte - appuyée par un dépouillement d'archives et la conduite d'une cinquantaine d'entretiens semi-directifs - nous permet de reconstituer les trajectoires des membres des trois groupes ayant le plus pesé sur l'institutionnalisation de la CIMIC en France. Les deux premiers sont en compétition pour le leadership des activités civilo-militaires au sein des armées tandis que le troisième constitue le soutien non militaire le plus constant à l'émergence du « projet ». Le premier groupe (les forces spéciales) est constitué de treize membres du bureau «affaires civiles » du Commandement des opérations spéciales (COS) créé en 1992. Ce groupe défend une vision dite « extensive » et " politique » appelée « affaires civiles » qui s'inspire des modes opératoires américains : l'objectif est la promotion des intérêts nationaux dans le cadre d'une intervention. Pour le deuxième groupe (les héritiers de l'armée d'Afrique), nous nous référons à un panel de dix officiers supérieurs et généraux de l'armée de terre. La vision dominante du projet civilo-militaire y est dénommée " actions civilo-militaires » (ACM). Qualifiée de « restrictive » et «militaro-centrée », cette vision ne s'interdit pas de participer à la promotion de l'influence nationale mais n'en fait pas une priorité : la finalité est d'œuvrer à l'acceptation de la force auprès de la population locale. Enfin, le dernier groupe (les urgentistes du ministère des Affaires étrangères [MAE]) est composé de sept acteurs ayant gravité des années 1980 aux années 2000 au sein des organisations du MAE dédiées à l'urgence (secrétariat à l'action humanitaire, cellule d'urgence, mission interministérielle pour l'Europe du Sud-Est). Ignorant les rivalités entre organisations militaires, les membres de ce groupe ont soutenu la gestation de la Coopération CiviloMilitaire afin de décloisonner leurs activités au sein de leur ministère. En se plaçant de leur point de vue, et en gardant à l'esprit le fait que le MAE, leur ministère de tutelle, appréhendait avec une extrême méfiance la possibilité d'une gestion civile des crises par les militaires, on pourrait quelque peu transformer l'adage : les adversaires (officiers affaires civiles du COS ou ACM de l'armée de terre) de mes adversaires (autorités des secteurs «diplomatiques » du MAE) deviennent mes alliés (à moi, urgentiste du MAE).

10. «La coopération civilo-militaire (CIMIC) désigne la fonction destinée à favoriser l'intégration de la force dans son environnement humain en général et auprès des populations locales en particulier, afin de faciliter l'accomplissement de sa mission, le rétablissement d'une situation sécuritaire normale et la gestion de la crise par les autorités civiles (administration, action humanitaire, reprise économique...) ", Coopération civilo-militaire, Doctrine interarmées, DIA-3.10.3(A) CIMIC(2012), No174/DEF/CICDE/NP du 17 juillet 2012, p. 19. 


\section{Les forces spéciales à la conquête des marchés de la reconstruction}

Parmi les leçons tirées par les autorités politiques et militaires françaises de la participation aux opérations dans le Golfe en 1991, la frustration économique liée à l'absence d'anticipation de l'après crise va présider au positionnement d'un groupe d'officiers des forces spéciales. Créé dès 1992, le bureau « affaires civiles » du COS a pour mission de faciliter l'accès à de nouveaux marchés pour l'industrie nationale tant au sein de pays dits en " crise » que dans les pays «émergents », en mettant à profit la présence d'une force militaire. L'objectif affiché est l'influence nationale à travers l'économie. La diffusion d'une vulgate militaro-industrielle sur le « retour sur investissement », le « prix du sang » et « l'intelligence économique » au cours des années 1990 est l'une des conséquences visibles de la montée en puissance de ce secteur d'activités ces dernières décennies. Comment ces pionniers ont-ils réussi à se voir confier le développement du projet civilo-militaire ? Quelles sont les caractéristiques organisationnelles du bureau «affaires civiles » ? Qui sont, d'où viennent et comment travaillent ses membres?

\section{L'importation du modèle américain}

Le modèle américain des Civil Affairs est perçu comme politique du fait de ses objectifs : soutenir le déploiement des forces en favorisant leur acceptation par la population et mettre en place des relais administratifs favorables aux intérêts américains. Quatre catégories d'enseignements vont être tirés de ce modèle par les officiers français qui viennent de l'observer de près au Koweï : l'existence d'une organisation Civil Affairs rattachée aux forces spéciales ${ }^{11}$, le découpage des compétences entre spécialistes et généralistes ${ }^{12}$, le recours à une majorité de réservistes ${ }^{13}$ et l'importance des expertises prédécisionnelles ${ }^{14}$. Pour qui cherche à comprendre pourquoi le «dossier » civilomilitaire est initialement revenu au COS dès sa création en 1992, trois pistes se combinent. La première a pour elle une logique fonctionnelle. Neuve, non conventionnelle, orientée autour du renseignement et de l'intelligence économique, jouissant d'un a priori fantasmatique, le COS serait donc « la » structure pour innover et expérimenter avec une discrétion maximale : unités polyvalentes particulièrement bien entrainnées et équipées, réservistes possédant de hautes qualifications civiles et rapidement mobilisables. Le COS est en effet la

11. 96th Battalion sous l'autorité du US Army Civil Affairs and Psychological Operations Command.

12. Le rôle des généralistes est de conseiller le chef militaire tandis que les spécialistes sont répartis en quatre sections : gouvernement (administration, santé et sécurité, système judiciaire, éducation, finances...), économie/commerce (politique monétaire, fiscale et budgétaire, logistique, agriculture et alimentation...), infrastructures (communications, transports, travaux, gaz, eau, traitement des déchets...) et fonctions particulières (culture, information, réfugiés, services d'urgence, environnement...).

13. $97 \%$ des effectifs sont formés de réservistes.

14. Elles visent à obtenir les renseignements concernant une zone d'intervention avant la projection des premiers éléments. 
seule unité militaire à dépendre directement du chef d'état-major des armées, et par extension du président de la République, chef des armées. La chaîne décisionnelle, (président de la République/chef d'état-major des armées/commandant de l'opération) est raccourcie au maximum. La deuxième piste nous oriente autour des avantages (re)distributifs entre organisations militaires dans le cadre des réformes. Le COS est l'organisation qui aurait « levé la main le plus haut ». Se voir confier de nouvelles missions, malgré l'inflation ambiante, devient un excellent moyen de justifier de besoins budgétaires et humains supplémentaires ou, à défaut, de limiter les pertes. Enfin, la dernière hypothèse, développée dans la dernière section de cette partie, est une " histoire d'hommes » et de réseaux.

\section{Les " affaires civiles » ou la mise en ceuvre d'interfaces militaro-industrielles}

En avril 1994, dans le cadre de la résolution prévoyant la reconstruction de Sarajevo, le bureau « affaires civiles » dépêche une quinzaine de réservistes et de soldats d'active en vue de fournir une expertise initiale de théâtre (EIT) visant à établir les priorités d'action sur la situation de la zone d'intervention autour de sept domaines : la santé, l'éducation, la culture, l'énergie, le transport et la privatisation pour un coût estimé à 75 millions de francs auxquels s'ajoute le désenclavement de Sarajevo évalué à 75 millions de francs supplémentaires. L'objectif est de faciliter le partage d'informations entre industriels, militaires et diplomates à deux niveaux : celui du lobbying auprès des grandes administrations et fédérations d'entreprises parisiennes et celui des opportunités locales dans le cadre des opérations de reconstruction. Au niveau central, l'association Études et actions d'urgence (EAU) fondée en novembre 1994, réunit des conseillers au commerce extérieur, des fonctionnaires des principaux ministères régaliens et quelques directions de groupes privés, avec le soutien du Mouvement des entreprises de France (Medef International). En janvier 1996 est fondé le groupe «Procure », en lien avec certains officiers «affaires civiles » et les responsables de la Fondation pour les études de défense (FED) ${ }^{15}$. Au niveau local, des réservistes du COS créent le même mois la Chambre de commerce et d'industrie franco-bosnienne. En relation avec une quinzaine d'entreprises françaises et une trentaine d'entreprises bosniennes, co-présidée par Sofinfra 16 et l'entreprise locale ZGP, la création de cette chambre de commerce se justifie alors par l'inexistence de poste d'expansion économique à l'ambassade et par l'absence de volonté de la direction des relations économiques extérieures (DREE) du ministère de l'Économie et des Finances ${ }^{17}$. Bénéficiant des services d'un réserviste COS déployé dès 1994, le

15. Voir la collection Cabiers de la FED : «Bosnie, les leçons ", nº 6, février 1997 ; «Intelligence, économie et défense », $\mathrm{n}^{\circ} 7$, avril 1997 ; «La montée en puissance du renseignement économique », $\mathrm{n}^{\circ} 8$, juillet 1997, "Stratégies économiques en Bosnie-Herzégovine », n 9 octobre 1997 ; «Des forces spéciales aux affaires civilo-militaires ", n 10, octobre 1997 ; «La gestion des sorties de crise. Actions civilo-militaires et opérations de reconstruction », $\mathrm{n}^{\circ} 12$, mars 1998.

16. Bureau d'études d'Aéroports de Paris (ADP). 
groupe Compagnie générale des eaux a remporté un marché de 6 millions de francs pour la gestion des canalisations de Sarajevo par l'intermédiaire de sa filiale SOGEA. Renault Véhicule Industriels, en lien avec son directeur des ventes militaires et lieutenant-colonel de réserve au COS, a obtenu sur fonds européens un marché de 12 millions d'euros par l'intermédiaire de sa filiale slovène. Société d'engineering de la SNCF pour l'étranger, la société Sofrerail, disposant elle aussi d'un officier réserviste au COS, a assuré la remise en état de la voie ferrée Ploce-Sarajevo ${ }^{18}$.

La réhabilitation de l'aéroport de Sarajevo est considérée comme un cuisant échec. Les 27 et 28 juin 1992, François Mitterrand est le premier président occidental à se rendre à Sarajevo depuis l'éclatement du conflit. La rénovation est un projet mûrement réfléchi par Sofinfra, qui a pris soin d'associer les autorités bosniaques, et a été conçue avec les acteurs identifiés par les EIT (International Management Group, bureau du Haut représentant de l'ONU, direction générale de l'aviation civile, DREE, ambassadeur de France à Sarajevo). Or, les marchés remportés y seront dérisoires au regard de ceux gagnés par des nations n'ayant peu ou pas du tout contribué à l'effort militaire ${ }^{19}$. Dans un autre domaine, le centre culturel André Malraux à Sarajevo, ouvert en 1995, est né de l'initiative de Francis Bueb, ancien directeur des Rencontres culturelles de la Fnac et du soutien du chef du bureau « affaires civiles ». Disposant d'une bibliothèque, d'une vidéothèque et de fonds documentaires sur la guerre, sa mission est de promouvoir la culture française : création du Festival du film de Sarajevo, rencontres européennes du livre, soirées consacrées aux auteurs de la région, financement de traductions littéraires, enseignements de français.

17. Défourneaux M., «Stratégies économiques en Bosnie-Herzégovine ", Cabiers de la FED, 9 1997 ; «La France en Bosnie : la désertion économique », Le Casoar, 148, 1998.

18. Daho G., "Les réseaux militaro-industriels français et la prospection des marchés de la reconstruction en ex-Yougoslavie ", Les Champs de Mars, 22, 2011, pp 133-151.

19. Cet épisode sera abondamment commenté : "Comment expliquer dans ces conditions que l'aéroport de Sarajevo ait été entièrement reconstruit, les entreprises autrichiennes ayant fourni les camions, les Suédois ayant vendu les moyens de déneigement, les Allemands le matériel aéronautique, et les Néerlandais les radars ? Reconstruit par des entreprises européennes, l'aéroport de Sarajevo a en réalité été financé par des fonds européens... et inauguré par le secrétaire d'État américain. Au total, malgré la remarquable expertise réalisée en mars 1993 sur le site de l'aéroport par le Commandement des opérations spéciales (COS) rien n'est revenu à une entreprise française alors que la gestion de l'aéroport et de l'espace aérien de la Bosnie-Herzégovine est confiée aux militaires français depuis 1992 ! », Gaïa R., Rapport d'information sur l'action civile des armées sur les théâtres extérieurs, commission de la Défense nationale et des forces armées de l'Assemblée nationale, 20 juin 2001, La Documentation française, p. 96. 
De façon schématique, on pourrait dire que le bureau "affaires civiles » du COS fait travailler ensemble des militaires fortement « civilianisés » (amiraux régulièrement au contact des hautes autorités politiques, des circuits économiques et des réseaux universitaires, officiers routinisés aux contacts avec les civils en opérations extérieures et à Paris) et des civils fortement « militarisés» (industriels et cadres d'entreprises proches de l'univers du renseignement). Au moment de la création du COS en 1992, la plupart d'entre eux se connaissent depuis une bonne décennie ; ils ont déjà travaillé ensemble à la direction générale de la sécurité extérieure (DGSE) au sein des services d'espionnage industriel. L'organisation du bureau peut être décrite en trois cercles concentriques. Le premier de niveau politico-stratégique est formé d'amiraux constituant aujourd'hui encore de véritables figures tutélaires. Dans la mémoire institutionnelle, les amiraux Pierre Lacoste 20 et Jacques Lanxade 21 font figure à la fois de pionniers et de modèles d'excellence. Un second cercle de niveau stratégique, à l'image par exemple des généraux Maurice Lepage et Jacques Saleun, créateurs du COS, ou des officiers de grades subalternes, chefs successifs du bureau, est composé de légionnaires socialisés aux milieux civils et spécialisés dans le renseignement. Enfin, au niveau opérationnel, le dernier cercle regroupe des réservistes hautement qualifiés, cadres issus des milieux industriels, chargés de cibler les secteurs-clés de la reconstruction via les EIT. C'est avant tout le multipositionnement de ces acteurs, à cheval entre les secteurs militaires et industriels, qui constitue la principale ressource engagée dans la tentative de formalisation «à l'américaine » des activités civilo-militaires. Ce n'est pas tant que ces acteurs appartiennent à différents univers mais plutôt que le cœur de leur travail consiste à amener ces différents univers à « se parler ». La plupart des interfaces militaro-industrielles créées par l'entremise de la nébuleuse COS en Bosnie seront réactivées au Kosovo à partir de 1999 : EAU devient Action d'urgence et développement (AUDE), " Procure ", «Procure 2 ».

"Q- Tout commence en mars 1999, avec le fameux serment du jeu de paume...

"Oui, serment du jeu de paume que j'ai facilité et qui s'est déroulé dans la salle du conseil d'administration de Schneider dans le soussol à Boulogne et où j'ai réuni tout le monde...

"Q-Donc il n'y avait pas, cette fois-ci, que des administrations?

«Non, j'ai réuni tout le monde...c'est-à-dire que j'ai fait ce que l'administration aurait dû faire.

20. Président de la Fédération des professionnels de l'intelligence économique depuis 2006, ancien directeur général de la DGSE de 1982 à 1986 et chef du cabinet militaire du Premier ministre Raymond Barre.

21. Ancien chef d'état-major de l'Élysée (1989-1991), le chef d'état-major des armées Jacques Lanxade (1991-1995), nous est présenté comme la figure de l'« officier diplomate ». Lanxade J., Quand le monde a basculé, Paris, Nil, 2001. 
«Q-Et qui convoquez-vous?

«Tous ceux que je connaissais... que je présumais être à la hauteur pour lancer un truc un peu coercitif...

«Q- Vos réseaux de Bosnie?

«Pas seulement... ceux que j'ai connus même avant la Bosnie, en Europe centrale. J'ai donc réuni des patrons d'entreprises, des gens de l'EMA [état-major des armées], du COS... il y avait des gens du COIA [Centre opérationnel interarmées], du cabinet du ministre, les gens de Bercy...»

(Entretien avec un ancien réserviste « affaires civiles » du COS, 12 février 2010)

Corollaire du multipositionnement de ces acteurs : la fongibilité des capitaux. En effet, au début des années 2000, alors que les marchés de la reconstruction au Kosovo sont déjà « distribués », certains vont fonder leur société de consulting en gestion de risque 22 ou d'intelligence économique ${ }^{23}$. Les espions industriels des années 1980 sont les forces spéciales chargées de prospective économique dans les années 1990 et deviendront, pour la plupart, les créateurs et capitaines d'entreprises nationales d'intelligence économique florissantes dans les années 2000.

«Alors la société que j'ai créée... on peut dire, si vous voulez qu'elle provient de cette époque... du constat que j'évoquais à l'instant sur l'ex-Yougoslavie, qui est pour moi un élément fondateur. Quand on a monté les premiers... "audits de théâtre”, on a donc propulsé avec nous sur le terrain des officiers de réserve, qui représentaient les grands groupes français, tous intéressés à la reconstruction du pays, donc BTP, énergie, environnement, téléphonie, etc. Ils nous avaient donné trois éléments explicatifs, trois raisons majeures pour lesquelles nous n'étions pas, nous Français, meilleurs dans la conquête des marchés émergents des pays sortant de crise ou de guerre :1) le défaut d'informations ; 2) le défaut de réseaux, donc d'influence en fait...; 3) le réseau de sécurisation. Donc quand on prend ces trois items, ce sont les éléments fondateurs de ma société aujourd'hui. En fait, en réalité, moi je fournis de l'analyse pays, et du renseignement économique, ça c'est pour répondre à la première batterie de questions. Je fournis de l'influence au sens lobbying, diplomatie parallèle et communication d'influence, ça c'est pour répondre au deuxième item et puis je fournis du conseil en sûreté, protection du patrimoine des entreprises. Donc effectivement ma société découle

22. C'est le cas du lieutenant-colonel à l'origine de la création des associations EAU/AUDE. Il a créé une société spécialisée dans la «prévention des risques et la gestion des crises ».

23. Un colonel a fondé une société d'intelligence économique, tandis que le cocréateur de la Chambre de commerce et d'industrie franco-bosnienne était, en 2005, le directeur général d'une société du même secteur basée en Europe de l'Est. 
du COS, du bureau des actions d'environnement ou d'influence du COS, c'est évident. »

(Entretien avec un ancien colonel « affaires civiles » du COS, Paris, 13 octobre 2009)

\section{Les héritiers de l'armée d'Afrique et le retour de l'action}

Entre 1994 et 2002, les officiers du COS se font progressivement déposséder de leur monopole par ceux de l'armée de terre qui, après des décennies d'immobilisme face au front de l'Est, s'enthousiasment à l'idée du « retour » de l'action. Au niveau bureaucratique, le chef d'état-major des armées nomme en 1994 un groupe de travail civilo-militaire chargé de produire les textes institutionnels (directive, concept, doctrine) sous le leadership d'organisations terriennes. Au niveau opérationnel, les premières unités spécialisées sont déployées en Bosnie en 1996 et au Kosovo en 1999 où les actions civilo-militaires (ACM), d'abord synonymes d'action humanitaire, vont être réorientées vers des objectifs d'influence. Pour disqualifier puis réintégrer les objectifs d'influence soutenus par le COS, les officiers de l'armée de terre disposent de ressources collectives : au multipositionnement de leurs homologues des forces spéciales, ils opposent un parcours d'excellence au sein de l'institution militaire qui leur permet de stigmatiser les dérives potentielles du mercantilisme des « affaires civiles » 24 .

\section{L'apprentissage de l'interposition bumanitaire}

L'armée de terre met en place ses unités ACM à partir de 1996. Trois formes de contrainte sont évoquées pour expliquer l'émergence de cet outil au niveau des «forces conventionnelles » : la multinationalisation, la juridicisation et l'humanitarisation des opérations de maintien de la paix ${ }^{25}$. Si l'on peut comprendre les avantages que le recours au multilatéralisme procure (partage des coûts et des risques, division du travail, gains de légitimité tirés des mandats internationaux, transferts de compétences et d'expérience entre alliés, etc.), le poids des institutions internationales agit aussi comme contrainte sur

24. Parmi les consignes données aux premiers officiers ACM de l'armée de terre, les formules comme «Surveillez-moi les cow-boys du COS ! » ou encore «Remettez-moi les lunettes noires à leur place! » sont abondamment citées par nos interlocuteurs.

25. En entérinant le passage de relais de l'ONU à l'OTAN en ex-Yougoslavie, les accords de Dayton signés en décembre 1995 scellent l'entrée dans la «troisième génération » d'opérations de paix. Trois types de transformations majeures semblent expliquer ce changement après 1995. D’abord, les cadres juridiques évoluent, du «maintien » vers « l'imposition de la paix » dans le cadre de l'application du chapitre VII, article 42, de la charte des Nations unies. En conséquence, l'amélioration des appareils coercitifs laissés à disposition des unités déployées, et surtout la souplesse dans leur possibilité même d'emploi, augmente la marge de manœuvre des organisations militaires. La troisième évolution notable concerne l'anticipation des dimensions administratives, politiques et économiques de la reconstruction d'après crise. Prévot-Forni E., «L'identité militaire à l'épreuve des opérations extérieures à finalité pacificatrice », Les documents du C2SD, 2001. 
l'emploi des forces car il nécessite des efforts de qualification et d'insertion du personnel et une recomposition des chaînes décisionnelles. Les alliés de l'intervention sont les concurrents de la reconstruction dans une multitude de domaines : diplomatiques (contrôle des ressources, encadrement administratif, influence), stratégiques et militaires (gestion de zones, implantation de bases militaires, contrôle des aéroports et de l'espace aérien, des ports et de l'espace maritime), économiques (circuits financiers et parts des marchés de la reconstruction), communicationnels (euphémisation de la présence armée, collaboration avec les ONG et les médias), culturels (influence linguistique, lycées, réseaux universitaires, centres culturels) et juridiques (droit anglosaxon contre droit romain, code des marchés publics, règles institutionnelles). Pour les unités projetées, la juridicisation se traduit par tout un corpus de « règles d'engagement » dont la finalité est d'encadrer l'usage de la force. C'est ainsi qu'émergent un certain nombre de symptômes du « soldat de la paix ». D'abord l'ouverture du feu est dramatisée ; ensuite, on constate une préférence tactique pour l'évitement du combat; enfin la figure du «soldat diplomate » émerge à tous les échelons hiérarchiques : il faut négocier pour passer un check point, escorter clandestinement un convoi humanitaire et ruser pour transporter des personnalités dont il faut anonymement assurer la sécurité. L'appréciation de l'usage minimal de la force en cas de riposte devient « le degré de force requis pour résoudre un conflit dans une situation donnée sans poser de problèmes à l'autorité responsable de l'emploi de cette force 26 ». Enfin, trois grands types d'actions humanitaires sont observables. Le premier consiste dans l'aide logistique fournie aux ONG pour des travaux de réhabilitation au profit des populations civiles. L'escorte de convois humanitaires et les opérations de déminage sont aussi un mode d'action fréquent. La « winterisation ", anglicisme désignant la livraison de matériel à l'approche de l'hiver, renvoie à l'ensemble des actions menées au profit des populations locales, généralement à l'échelle d'un quartier ou d'un village : distribution d'eau, de vêtements, de matériels de chauffage, assistance médicale, ramassage des ordures, réparation de voiries et de réseaux de gaz et d'électricité ${ }^{27}$.

Cette historiographie institutionnelle est pourtant trompeuse : la transformation des doctrines et des organisations militaires correspond sans doute moins à une logique d'adaptation à l'environnement stratégique que d'innovation et d'improvisation des chefs militaires. Les actions humanitaires n'ont pas véritablement de dénominateur commun si ce n'est de favoriser l'acceptation des forces en devançant les sollicitations de la population. La mise en œuvre décentralisée des ACM repose sur l'initiative des chefs.

26. Thièblemont A., «Expériences opérationnelles dans l'armée de terre : unités de combat en Bosnie (1992-1995) », Les documents du C2SD, 2001.

27. Braëm Y., Géopolitique des relations militaires-humanitaires : comparaison des interventions au Kosovo et en Afghanistan, thèse de doctorat en géopolitique, Université Paris 8, sous la direction de Béatrice Giblin, 2007. 
« Mais il y avait quand même un problème, c'est que j'avais plein de traîne-savates qui s'astiquaient toute la journée à la vodka locale et qui à 10 heures du matin étaient capables de monter à l'assaut, en baskets dans la neige, en gueulant comme des nazes et en en prenant plein la gueule. [...] Donc j'ai été voir les autorités politiques locales et je leur ai demandé : "Mais pourquoi vous ne faites pas quelque chose ? Vous avez des brasseries.” Et les gars m'ont dit “oui, mais on a plus rien pour faire des capsules”. C'est vrai qu'à l'époque les mecs récupéraient même des fermetures éclairs pour faire des balles. “Alors vous avez besoin de combien ?" "Cinq tonnes." J'ai dit : “D'accord, je ramène, je gère le stock et je distribue à la demande.” Et quand les mecs se sont mis à boire de la bière, ils devenaient moins agressifs qu'avec la vodka. »

(Entretien avec un ancien chef du bureau ACM, 1996, Paris, 16 novembre 2009)

Le commandant de la zone de Bihac se soucie de l'intégrité des forces en opposition qu'il est censé observer. Sa mission consiste avant tout à les empêcher de livrer combat plutôt qu'à les mettre hors d'état de nuire. En s'adressant à la brasserie et en proposant d'en devenir le fournisseur, le colonel agit comme une véritable entreprise de services publics sans en avoir les prérogatives formelles. Le but de l'opération est caractéristique des objectifs limités que se fixent les chefs : ici, en substituant la bière à la vodka, il s'agit d'abaisser le niveau de violence ${ }^{28}$. L'apparition du civilo-militaire sous sa forme humanitaire en Bosnie s'apparente pour certains officiers à un processus de « redécouverte de l'eau chaude ». Le caractère totalement «bricolé » de celle-ci est assumé sans aucun détour. Par ailleurs, ces initiatives semblent presque répondre à des objectifs davantage "récréatifs » qu'opérationnels. Elles visent à « oxygéner » les hommes, leur changer les idées, les sortir de la torpeur de l'interposition. C'est ce que certains nomment le «syndrome Kouchner 29 ».

\section{Les actions civilo-militaires, entre soutien aux forces et influence}

Les dispositifs ACM sont progressivement standardisés à partir de 1999 en Bosnie et au Kosovo : au niveau " opératif », c'est-à-dire dans la capitale de la zone d'intervention (Sarajevo, Pristina), les unités civilo-militaires veillent à soutenir l'influence nationale en conseillant la plus haute autorité militaire française au sein de l'état-major multinational de l'OTAN, tandis qu'au niveau «tactique », là où sont positionnés les bataillons français (Mostar, Mitrovica), elles s'emploient à favoriser l'acceptation locale des forces. On

28. L'ex-Yougoslavie regorge de récits héroïques sur les chefs face à la logique sclérosante de l'interposition : dans un tout autre domaine que la CIMIC, voir « l'épisode la soupe » relaté par le colonel Goya dans sa mission anti-sniping, Goya M., " Une expérience de lutte contre les snipers ", Inflexions, 3, 2006, p. 97-112.

29. Thièblemont A., art. cit., 2001, p. 104. 
constate donc une sorte de bipolarisation des ACM conventionnelles entre l'option restrictive et militaro-centrée (le soutien à la force) et l'option extensive et politique (l'influence nationale). Au niveau opératif, la cartographie des partenaires institutionnels et des infrastructures du pays est la priorité d'action. Le chef du bureau ACM oriente la collecte, l'exploitation et la diffusion des informations auprès du détachement de niveau tactique et entretient des contacts réguliers avec les acteurs nationaux ou internationaux. Les cellules liaison, expertise et coopération technique ouvrent à favoriser le placement d'officiers français auprès des OI et des administrations locales. Le but formel de l'insertion est moins de nommer des représentants nationaux à des postes de décision, que des spécialistes à des postes intermédiaires où se jouent l'accès à l'information et la mise en forme des options. À travers les principales organisations visées par l'insertion ${ }^{30}$, nous pouvons déduire les domaines prioritairement investis : la multinationalisation du civilo-militaire, la gestion des mouvements de réfugiés et la recherche de financements, si possible européens. Au Kosovo, où les dispositifs d'insertion se densifient, on constate que la collecte de fond devient l'activité la plus consommatrice en personnels. Les unités de l'armée de terre ne disposant pas de budget propre, tout projet d'envergure nécessite non seulement la recherche d'aides financières auprès de bailleurs, mais aussi d'entreprises locales, d'OI ou d'ONG. Au niveau tactique, le détachement ACM est composé de deux cellules d'expertise et de recherche de financement, d'éléments de liaisons et d'équipes veillant au suivi des projets qui prennent la forme d'initiatives peu coûteuses, rapides à mettre en œuvre et axées sur l'amélioration des conditions de vie des populations. La finalité demeure, avant l'influence, l'acceptation des forces et la recherche de renseignements sur les mouvements des belligérants et l'état d'esprit des populations.

\section{L'béritage, le parcours d'excellence et la socialisation aux milieux civils}

Tout comme leurs homologues des forces spéciales, la plupart des officiers de l'armée de terre se saisissant du projet ACM - ou se faisant « embarquer » dedans - entre 1996 et 2001 se connaissent bien. Certains ont été les premiers chefs ACM projetés dans l'urgence ou des commandants de zone ayant pris l'initiative de recourir à ce type d'actions. D'autres, au niveau des états-majors centraux, se sont intéressés de près au « dossier », qu'ils aient été chargés de sa formalisation conceptuelle, de le porter ou de le faire connaître aux acteurs non militaires ou qu'ils se soient « retrouvés » en position d'arbitrage. Saintcyriens, brevetés de l'école de guerre, ayant tous cumulé des responsabilités en états-majors centraux et en opérations extérieures, les membres de notre panel jouissent d'un parcours d'excellence lié aux choix de spécialisation : les armes de mêlés et l'infanterie, bénéficiant, parce que directement liés au combat,

30. Coalition Joint CIMIC Task Force, Office of the High Representative, Haut-Commissariat aux réfugiés, Banque mondiale, Organisation pour la sécurité et la coopération en Europe, International Management Group. 
d'un prestige élevé au sein des armées. Ils sont issus des rares troupes déjà professionnalisées dans les années 1980, celles de l'armée d'Afrique, qui «sortent » le plus à cette époque : dans $80 \%$ des cas, il s'agit d'officiers ayant choisi leur première affectation dans les troupes de marine ou la Légion. L'armée d'Afrique désigne les unités militaires engagées dans la pacification coloniale : la Légion étrangère, les troupes de marine et les troupes indigènes. À l'opposition entre l'armée coloniale et l'armée continentale a succédé durant la guerre froide la confrontation entre l'armée d'Afrique et l'armée de l'Est. L'héritage de cette confrontation constitue encore aujourd'hui un marqueur identitaire très important ${ }^{31}$. La doctrine dissuasive tend à consacrer le rôle de la marine et de l'armée de l'air dans la perspective d'une frappe nucléaire. A contrario, ces officiers regrettent que « l'action » soit jetée aux oubliettes.

«Quand nous sommes arrivés avec ces idées... des idées d'actions, qui étaient à la fois militaires, de maintien de l'ordre mais aussi de contact avec la population... moi j'ai entendu ça toute ma vie. Donc on arrive et on nous dit: "on ne parle pas politique, de toute façon vous la fermez, il y a le nucléaire, les Russes en face de nous et de toute façon s'ils attaquent, on tire une bombe atomique." On a rapidement compris que si on tirait avec une bombe atomique, on ne voyait pas bien l'intérêt de s'entraîner avec des chars. Donc nous avons fait semblant pendant des années. »

(Entretien avec un général, légionnaire, dans un café, Paris, 29 juin 2009)

Dans les années 1980, ils vont ensuite être projetés sur des théâtres, notamment au Liban, en Nouvelle-Calédonie et au Tchad, dont les enjeux sont proches de ce que deviendront les opérations de maintien de la paix. Il s'ensuit une sorte de refus farouche de l'immobilisme du front de l'Est, qui se traduisait certes déjà par leur préférence pour l'armée d'Afrique, mais qui est maintenant entretenu par leur mobilité professionnelle. Au moment du rééquilibrage dissuasion/action, ils constituent le seul réservoir de compétences disponibles. Le jeune capitaine envoyé au Liban au début des années 1980 deviendra souvent, après son passage à l'école de guerre, le colonel responsable des actions civilo-militaires en ex-Yougoslavie dans les années 1990. Au-delà du parcours d'excellence et du monopole de l'expérience des interventions, leurs mobilisations autour du projet civilo-militaire est favorisée par leur très forte socialisation aux milieux civils, au niveau tant des organisations centrales (décideurs politiques, milieux diplomatiques, industries de l'armement, réseaux universitaires) que des opérations extérieures (population locale, armées alliées, ONG, OI). Nous avons confirmation que $60 \%$ du panel possède une qualification de niveau master (histoire, sciences de l'infor-

31. Daho G., «L'érosion des tabous algériens. Une autre explication de la transformation des organisations militaires en France ", Revue Française de Science Politique, 64/1, 2014, pp. 5778. 
mation et de la communication, science politique, langues étrangères) et $20 \%$ de niveau doctorat (histoire, sciences de l'information et de la communication) à une époque où les passerelles avec les milieux académiques n'étaient pas institutionnalisées. Au début de l'année 2010, si 40 \% d'entre eux sont encore en activité au sein des armées à des postes à responsabilité (gouverneur militaire de Paris, directeur du Musée de l'armée, commandant de la force d'action terrestre, chef de projet au Centre interarmées du concept, de la doctrine et de l'expérimentation), $60 \%$ se sont reconvertis dans la haute fonction publique nationale (Conseil d'État, chargé de mission Lille) ou internationale (International Management Group, Organisation pour la sécurité et la coopération en Europe) et dans le privé (Gaz de France Suez, groupe DEMOS).

\section{Des alliés improbables aux Affaires étrangères : les urgentistes}

Les officiers du COS et les héritiers de l'armée d'Afrique vont disposer au Kosovo d'un relais institutionnel assez inattendu : celui des urgentistes du ministère des Affaires étrangères qui, empêtrés depuis le milieu des années 1990 dans la réforme de la coopération et du développement au sein de leur propre ministère ${ }^{32}$, ont vu dans l'initiative civilo-militaire une occasion de décloisonner leurs propres activités. Ces alliances bureaucratiques revêtent pour le chercheur un caractère " contre-objectif » puisqu'elles mettent notamment en relation des héritiers nostalgiques des «batailles » et parfois même de l'«Algérie française » avec des militants des droits de l'homme ou de l'humanitaire à la sensibilité de gauche nettement affirmée.

\section{Des appuis politiques inédits}

Créée en juin 1999, sous l'impulsion de Jean-Maurice Ripert, conseiller diplomatique du Premier ministre Lionel Jospin, la mission interministérielle pour l'Europe du Sud-Est (MIESE) a pour président Roger Fauroux et se compose de quatre représentants de trois ministères (un diplomate et un coopérant pour les Affaires étrangères, un colonel de la Défense et un ancien directeur de la Direction des relations économiques extérieures du ministère de l'économie et des finances, DREE). Mandatée pour assurer la coordination interministérielle dans les Balkans, la MIESE a contribué aux résultats civilomilitaires français au Kosovo. Qu'il s'agisse de la diffusion des EIT directement auprès des entreprises, du " chapeautage ", non sans heurts, des structures informelles comme AUDE et Procure 2, de l'aide à l'insertion de spécialistes au sein des OI, de la tenue de réunions régulières avec les différents services ministériels concernés, de coopération décentralisée et de financement d'expertises, la MIESE a contribué à donner au(x) projets(s) civilo-militaire(s) une visibilité nouvelle. Parallèlement, autour de Bernard Kouchner, alors

32. Cypel S., «La réforme ! La réforme ! », Le Monde, 25 avril 2001 ; « La disparition de la "Coopé” ", Le Monde, 26 avril 2001 ; « Tout changer pour ne rien changer ? ", Le Monde, 27 avril 2001. 
représentant spécial de l'ONU au Kosovo dès le 30 juin 1999, le(s) projet(s) civilo-militaire(s) dispose(nt) désormais d'un relais local. Les dispositifs d'insertion au sein de la Mission intérimaire des Nations Unies pour le Kosovo (MINUK) se renforcent dans les secteurs de la réhabilitation d'infrastructure, les télécommunications, la fiscalité et l'agriculture. Les contacts entre les officiers « affaires civiles ", les membres de la MIESE et les principaux collaborateurs de Bernard Kouchner à la MINUK ont été fréquents ${ }^{33}$. Ce dernier a luimême souligné la qualité du travail de reconstruction effectué par les militaires ${ }^{34}$.

Ce sont surtout les conclusions du rapport final remis par Roger Fauroux au Premier ministre qui auront un impact fondamental sur l'institutionnalisation de la Coopération Civilo-Militaire. Elles ont d'autant plus surpris que si le MAE a « accepté » qu'il prenne la présidence de cette mission sur demande de Matignon, c'est parce qu'il était jusqu'alors considéré comme quelqu'un qui ne «ferait pas de vagues ». Ce rapport, désormais « introuvable » dans les administrations, fournit aux officiers impliqués dans la formalisation du projet, et au-delà de leur organisation d'appartenance, un argumentaire " clé en main » pour négocier avec les autorités politiques : insuffisance du volontarisme politique, coordination interministérielle déficiente, absence d'agence spécialisée dans la gestion de crise. En 2008, la plus haute autorité militaire de la chaîne CIMIC se montre toujours solidaire de ce triptyque :

"Q- Je voulais revenir, pour finir, sur la façon dont les responsables CIMIC critiquent le niveau politique...

«On n'a pas à critiquer le politique mais on peut donner son avis... on est quand même citoyen...

"Q- Tout à fait... et puis vous avez des besoins qu'il faut exprimer, et notamment vis-à-vis des politiques... Donc les trois critiques les plus récurrentes sont: 1) absence de véritable volontarisme politique traduit en état final recherché clairement édicté...

«Clairement énoncé... oui...

«Q-2) absence de coordination interministérielle...

«Ça c'est évident...

«Q-3) absence de guichet unique, d'agence centralisatrice...

«Ça ça dépend aussi, vous parlez pour la France?

33. Notamment, d'après nos entretiens, avec Éric Chevallier, ambassadeur de France en Syrie depuis 2009 et conseiller politique de Bernard Kouchner à la MINUK, et Bernard Salomé, directeur depuis 2008 de la Fondation du millénaire pour les financements innovants pour la santé, alors conseiller économique.

34. Il relaye ainsi un certain nombre de difficultés pratiques auxquelles le civilo-militaire naissant est confronté : stratégies de placement des experts et réservistes français au sein des OI insuffisantes, indépendantisme pénalisant des ONG françaises, lenteurs et rivalités bureaucratiques entre administrations, absence d'agence gouvernementale spécialisée comme en possèdent les Américains, les Allemands et les Britanniques. Kouchner B., Audition sur les actions civilo-militaires, commission de la défense nationale et des forces armées de l'Assemblée nationale, $\mathrm{n}^{\circ} 37,23$ mai 2001. 
«Q- Tout à fait, j'évoque les propos des officiers supérieurs de la CIMIC nationale...

«Oui, parce que ça correspond exactement à notre caractère gaulois... moi je vous l'avais dit, je suis pour l'interministériel, que le chef soit du MAE, de Bercy ou d'ailleurs, de la Défense... moi je m'en fous du moment que j'ai un bon chef et qu'il me donne des ordres. Moi je n'ai pas d'états d'âme, je préfère un chef qui donne des ordres plutôt qu'un chef de chez nous, un militaire, qui ne donne pas d'ordres... voilà... après bon vous avez des rivalités de pouvoir, tout ça. Mais c'est vrai qu'en France, au niveau interministériel, on est un peu plus compliqué que les autres pays. »

(Entretien avec un colonel de l'armée de terre, chef du bureau CIMIC au Centre de planification et de conduite des opérations, Paris, 26 mai 2008)

L'influence des conclusions du rapport se retrouve dans la nouvelle doctrine de 2005, ni « affaires civiles » ni « ACM » mais désormais CIMIC en langage OTAN. Celle-ci intègre « l'influence nationale » comme objectif mais maintient la priorité accordée au «soutien à la force ». L'arbitrage des « grands chefs 35 » vise à recentrer les usages sur le pôle restrictif du soutien à la force, craignant un certain nombre de dérives ( "l'officier n'est pas une ONG en kaki ou un VRP en uniforme »). Il traduit surtout un certain pragmatisme : faisons du soutien à la force faute de mieux... sans fermer la porte à un hypothétique volontarisme politique ou à l'investissement des autres ministères. D'un côté, l'option consistant à mettre en place de façon conjoncturelle une structure interministérielle légère autour d'un homme possédant un certain poids politique confirmé par mandat et composée de représentants des différents ministères régaliens semble une solution cohérente et flexible ${ }^{36}$. Elle permet de gagner du temps en raccourcissant considérablement les délais et les canaux de prise de décisions. En revanche, l'absence de structure permanente - à l'image des agences spécialisées de nos principaux alliés (USAID, DfID, GTZ ${ }^{37}$ ) peut être pénalisante en termes de constitution d'une mémoire institutionnelle, de coordination, de veille et d'anticipation, de recherches de financements, mais aussi d'aide à la décision. Les autorités du MAE estiment que la gestion civile des crises est traditionnellement de leur ressort ; celles du ministère de la Défense affirment, notamment pour des raisons sécuritaires, qu'elles seules possèdent le savoir-faire et la réactivité en dépit de budgets insuffisants.

35. Selon une formule consacrée par nos interlocuteurs désignant des positions institutionnelles et des qualités exceptionnelles. Il s'agit ici des généraux Bernard Thorette et Henri Bentégeat, respectivement chef d'état-major de l'armée de terre et chef d'état major des armées.

36. Cette « option» sera d'ailleurs reconduite en Afghanistan avec la mise en œuvre de la cellule AFPAK successivement dirigée par Pierre Lelouche et Thierry Mariani.

37. United States Agency for International Development (USAID) : Agence des États-Unis pour le développement international ; Department for International Development (DfID) : Département britannique du développement international ; Gesellschaft für technische Zusammenarbeit (GTZ) : agence allemande pour la coopération technique. 
Si l'on s'en tient aux formes d'interactions institutionnalisées entre la Défense et le MAE, la MIESE a clairement créé un précédent kosovar en court-circuitant les canaux habituels de prise de décision.

«L'expérience inédite de la coordination interministérielle d'une sortie de crise par une mission ad hoc, relativement autonome, a bousculé le fonctionnement traditionnel des administrations [...]. Le dispositif institutionnel, mis en place à la fin de la guerre du Kosovo, était inédit en France. C'était la première fois qu'une structure ad hoc, hors ministère, était chargée de coordonner l'effort français dans un contexte de sortie de conflit. Cette initiative du Premier ministre répondait avant tout à la volonté de mener une nouvelle forme de "diplomatie d'action", intégrant des acteurs privés dans une logique d'influence française. L'autonomie laissée à la MIESE dans ses initiatives sur le terrain fut parfois perçue au sein du MAE comme "incontrôlable". Au-delà de la dimension politique de la MIESE, sa mise en place et les relations privilégiées qu'elle a entretenues avec les militaires et les entrepreneurs français ont parfois court-circuité les initiatives "traditionnelles" de coopération. La cellule Balkans, au sein du ministère de la Coopération, était initialement chargée de gérer l'aide française en Albanie, en Macédoine et au Kosovo. Deux initiatives se sont en fait côtoyées sans véritablement collaborer : celle de Matignon, et la continuité civilo-militaire avec une orientation essentiellement économique, et celle du Cabinet du ministre délégué à la Coopération, impliquée dans les questions de financements et de mobilisation d'autres acteurs (ONG et collectivités locales). Il n'y a pas eu de réelle coordination et complémentarité entre la MIESE, la Coopération et le MAE. La MIESE et la CELLUR ont souligné l'aspect peu opérationnel et peu coopératif du Quai d'Orsay dans le contexte de postconflit du Kosovo. [...] Ces deux structures ont cependant salué le soutien de la Défense et des militaires français sur le terrain, qui ont accompagné leurs interventions au Kosovo. De son côté, le MAE a pointé du doigt le caractère désordonné et incohérent des initiatives menées sur le terrain. » 38

\section{Trajectoires de marginaux-sécants 39}

Après avoir intégré le MAE en 1973 et passé le concours interne avec succès en 1979, la future directrice financière de la cellule d'urgence du MAE et secrétaire générale de la MIESE démarre sa carrière dans la diplomatie en se spécialisant dans deux domaines marginaux : la promotion des droits de

38. Haut Conseil à la coopération internationale, (HCCI), rapport annuel d'activités, 2005, p. 72. 39. "C'est le pouvoir dit du "marginal-sécant" [...] un acteur qui est partie prenante dans plusieurs systèmes d'action en relation les uns avec les autres et qui peut, de ce fait, jouer le rôle 
l'homme (en tant que chargée de mission au MAE de 1984 à 1986, puis à la présidence de la République de 1986 à 1988) et la création de structures ad hoc liées à l'humanitaire d'urgence.

« La cellule d'urgence, c’est pareil... ça n’intéresse pas le Quai d'Orsay, tout ce qu'ils voyaient c'est... bon, je suis quelqu'un qui travaille, ça c'est assez reconnu, et qu'il fallait quelqu'un d'un peu solide... finalement c'est des sujets qui... moi je me suis toujours occupée de sujets qui n’intéressaient pas le Quai d'Orsay. Pas parce que c'est des sujets que j'ai choisis, mais parce que ça n'intéressait pas, et moi ça m'intéressait, à chaque fois... »

(Entretien avec l'ancienne directrice financière de la cellule d'urgence et secrétaire générale de la MIESE, Paris, 25 octobre 2009)

En 1978, son collègue et futur directeur opérationnel de la cellule d'urgence est envoyé à Tyr, au Liban, pour s'occuper d'un dispensaire après avoir créé l'antenne de Médecins sans frontières (MSF) à Dijon. À son retour en 1979, au moment de la scission du monde humanitaire ${ }^{40}$, il prend le parti de Bernard Kouchner avant que les deux hommes ne se séparent. En 1980, Bernard Kouchner crée Médecins du monde (MDM) tandis que de son côté, le futur directeur opérationnel met en œuvre Aide médicale internationale (AMI). C’est en mai 1988 qu'il rejoint Bernard Kouchner nommé ministre de la Santé et de l'Action humanitaire suite à la réélection de François Mitterrand. Le ministère dispose de deux outils : la direction générale de la coopération internationale et du développement (DGCID) et la délégation à l'action humanitaire (DAH). À l'image des réformes touchant le Royaume-Uni en 1997, les services dits de la « coopération », traditionnellement regroupés dans un ministère ou un secrétariat d'État «à part » ont été rattachés en $1998 \mathrm{au}$ MAE, leurs attributions et leur personnel étant désormais placés sous la responsabilité de la DGCID. De son côté, la DAH est le successeur du Service de l'action humanitaire (SAH) qui pilotait la cellule d'urgence du MAE dans les années 1990. L'enjeu pour les membres de la cellule d'urgence est de se rapprocher des coopérants :

« Cette cellule d'urgence, il faut voir ce que c'était... un vieux bâtiment en préfabriqué sans toilettes, rue Monsieur, ex-ministère de la Coopération... j'ai d'ailleurs réussi à le faire dire à Bernard [Kouchner] devant l'Assemblée nationale... [rires] il y avait trois agents. Voilà le service d'urgence de l'État français... ridicule... donc on demande, non pas une mise à disposition mais un rattache-

indispensable d'intermédiaire et d'interprète entre des logiques d'action différentes, voire contradictoires. ", Crozier M., Friedberg E., L'Acteur et le système, Paris, Le Seuil, 1981 [1977], p. 86.

40. En 1979, suite à l'opération « Un bateau pour le Viêt-Nam », le groupe des biafrais, pionniers de MSF, se divise entre les partisans de cette mission et ceux qui la jugent trop médiatique ou racoleuse. 
ment de la cellule au ministère, au SAH. [...]. À un moment Bernard me dit, à propos de la succession de l'ancien directeur qui avait été cité dans une affaire bizarre, “on t'impose”. J'ai dit d'accord. Et donc en interministériel on a demandé le rattachement de la cellule et ma nomination. Mais on n'a jamais rien vu venir...

«Q-Qu'est ce qu'il s'est passé?

"Moi ensuite je n'ai pas arrêté de me friter avec le directeur de cabinet, un énarque que Bernard a dû prendre, il n'y connaissait rien et on lui a dit de faire comme ça... et donc je disais à Bernard ne t'encarte pas, tu fais partie de la société civile, et deuxième point de conflit avec ce directeur de cabinet, on avait demandé le rattachement de la cellule d'urgence. Et lui ne voulait pas se mettre à dos le quai d'Orsay... alors qu'on était rattaché à Matignon... et devant Bernard, je lui dis que même Rocard [Premier ministre] était d'accord pour ce rattachement. Bernard ne comprenait rien à la différence entre une mise à disposition et un rattachement. Donc au final, il n'y aura pas de rattachement [...] Donc on est mis à disposition du SAH mais pas rattaché. Il était sous double tutelle, Affaires étrangères et Coopération et on fonctionnait plus volontiers avec les contrôleurs financiers de la coopération parce qu'ils savaient ce que c'était que la coopération, l'urgence. »

(Entretien avec l'ancien directeur opérationnel de la cellule d'urgence, Paris, 15 décembre 2009)

Ces logiques de rattachement institutionnel franchissent un cap : il ne s'agit plus de se «battre » pour obtenir le rattachement souhaité, il s'agit de ne pas se faire «tuer» par les collègues nommés à cette fin.

« Je suis donc nommé comme directeur opérationnel et on me refile dans les pattes un directeur financier qui se nomme [...] et qui était là pour me tuer... donc les choses n'avancent pas du tout...

"Q- Vous me dites [...] "a été nommée pour me tuer"... alors pourquoi ne l'a-t-elle pas fait? Il semble que cela se soit plutôt bien passé entre vous...

«Ah non, non, on s'est foutu sur la gueule...

«Q-Au Kosovo, ça semble fonctionner plutôt bien...

"Ah non mais ça c'est plus tard... au départ, on se met sur la gueule... elle dit A, je dis B...

"Q-Mais par rapport aux arcanes diplomatiques $d u M A E$, il semble qu'elle vous ressemble un peu...

"Oui, ça c'est sûr... mais à l'époque je ne le sais pas si vous voulez... mon réflexe c'est de ne pas la laisser me tuer... Elle, au bout d'un certain temps reconnaît la pertinence des missions montées qui prennent de l'ampleur et puis un jour on en arrive à discuter... l'ob- 
jectif n'est plus de se tuer, on travaille ensemble... mais c'est le fait que les opérations marchent, que l'on soit créatif et imaginatif, que l'impossible n'existe pas... on fait d'abord, on trouve le fric après... donc pendant deux, trois mois on s'est opposés, et puis au bout de six mois quand on a vu quelques succès comme en Arménie, on s'est dit que tout ça était ridicule... et que nos adversaires étaient plutôt au quai d'Orsay... c'est ça qui va nous rapprocher. Et puis elle avait une conception de l'administration plus profonde que moi, j'imaginais les opérations et c'est elle qui se démerdait avec les appels d'offre, les contrôleurs financiers... elle avait pas mal de complices au Quai d'Orsay... et plus tard, quand ils ont attaqué la cellule d'urgence, on l'a défendue tous les deux, bec et ongles jusqu'au bout. Donc c'est une amie. »

(Entretien avec l'ancien directeur opérationnel de la cellule d'urgence, Paris, 15 décembre 2009)

De son côté, la directrice financière confirme :

« Pour la cellule d'urgence du MAE Bernard Kouchner choisit [...] et le MAE me choisit car il ne faisait pas confiance dans les choix de Bernard... et on me dit "tu es là pour emmerder, enfin pour coincer, enfin pour être sûre qu'il ne fasse pas n'importe quoi en gros”. Donc voilà, quand Bernard nomme [...] à la cellule d'urgence, le Quai d'Orsay dit, "on acceptera cette nomination que s'il y a un co-directeur”...

"Q-Parce qu'on ne fait pas confiance dans les choix de Bernard Kouchner?

«On ne fait pas confiance à Bernard Kouchner...

«Q-Pourquoi?

«Parce que c'est une ONG [...]. À l'époque au Quai on me dit "comment tu peux t'occuper des ONG, ce sont tous des agents de l'Union soviétique”. »

(Entretien avec l'ancienne directrice financière de la cellule d'urgence et secrétaire générale de la MIESE, Paris, 25 octobre 2009)

\section{Solidarité défensive, transactions intersectorielles et réseaux socialistes}

Les urgentistes du MAE développent une solidarité défensive qui se densifie au cours des années 1990. Comme le dit le directeur opérationnel : «Nos adversaires étaient plutôt au Quai d'Orsay... c'est ça qui va nous rapprocher. » Les membres de la cellule sont la cible régulière de procédures d'inspection et de contrôle financier visant à condamner certains appels d'offre publics réalisés dans l'urgence entre 2000 et 2002 : pour les acteurs concernés, le MAE se vengerait ainsi de la «trahison » de Roger Fauroux. À partir de 
2002, le directeur opérationnel déplore la perte de ses «parapluies » dans le jeu institutionnel qu'il a notamment contrecarrée au moyen de certaines « transactions » avec les acteurs militaires :

"Sur cette affaire [Lors d'une visite officielle en Israël à la fin du mois de février 2000, le Premier ministre Lionel Jospin qualifie les attentats imputés au Hezbollah de "terroristes" et recevra des jets de pierres par une foule en colère], Jean-Maurice [Ripert] s'est fait doucement gicler... il a été nommé ambassadeur en Grèce et donc moi j'ai perdu mon parapluie... [la directrice financière] s'est fait gicler par [Charles] Josselin [secrétaire d'État à la coopération], j’ai perdu mon deuxième parapluie... ensuite Bernard [Kouchner] rentre et ne pouvait rien pour nous... Jospin était comme ça, chacun dans son coin, et au MAE ça enchaîne... l'administration du Quai se déchaîne sur nous, audit sur audit... ensuite il y a eu la tempête et puis l'Afghanistan... moi j'ai conseillé les militaires, j'ai passé des notes en sous main au général [alors chef du cabinet militaire du ministre de la Défense Alain Richard]... comme on avait bien travaillé avec le COS et les ACM à Mitrovica... »

(Entretien avec l'ancien directeur opérationnel de la cellule d'urgence, Paris, 15 décembre 2009)

En empruntant l'idéal-type du marginal-sécant ${ }^{41}$, on pourrait dire que le «pouvoir » des membres de la cellule d'urgence au sein du MAE tient au fait qu'ils ont le monopole de la gestion des relations de leur institution avec une partie de son environnement organisationnel (armées, ONG, sécurité civile...). À mesure que ce monopole se renforce, d'un côté ils suscitent la méfiance et de l'autre ils renforcent leur «pouvoir » au sein de leur institution.

«Q- Vous avez très tôt développé le sens du contact, notamment avec les humanitaires mais aussi les militaires. Est-ce qu'il s'agit d'une qualité que l'on vous reconnaissait déjà à l'époque?

«Enfin c'est un fait, je ne sais pas si c'est une qualité, parce que le Quai considère ça comme un problème... mais c'est un fait que j'ai un énorme contact avec les ONG, encore aujourd'hui d'ailleurs, il y avait les militaires aussi, parce que quand $j$ 'ai pris la cellule d'urgence, j'avais l'habitude de travailler avec eux et pour parler vulgairement, je ne leur avais jamais fait d'enfant dans le dos y compris sur les remboursements du Quai. Ça ça a été pour eux une preuve de loyauté, et comme je sais que c'est une administration où la mémoire ne se perd pas, par ailleurs, quand il y avait des sujets importants, je les ai toujours fait venir... je pense aux crises d'Afghanistan en 1997-1998, avec l'entrée des Talibans à Kaboul, on 
faisait sortir les ONG, je me souviens parfaitement, à une réunion, avoir fait venir des membres de la Défense parce que cela paraissait évident et je me suis faite engueuler... engueuler par le Quai d'Orsay. Pour moi l'interministériel est quelque chose d'important et d'utile et je fonctionne dans l'interministérialité, je ne sais pas faire autrement. Il ne faut pas oublier que j'ai fait un long passage à l'Élysée et quand je travaille pour le Quai d'Orsay, je travaille pour la France, je ne travaille pas pour le Quai d'Orsay... enfin je travaille pour le Quai d'Orsay, mais si c'est l'intérêt du Quai contre l'intérêt de la France, il n'y a pas photo pour moi. Et c'est peut-être là où je diffère de mes collègues...

«Q-Est-ce que finalement, vous n'êtes pas autant appréciée par les officiers supérieurs parce que vous avez cette autocritique sur le $M A E$ ?

«Oui.

«Q- On peut prendre appui sur une diplomate qui en plus sait taper sur sa propre maison quand il le faut?

"Non, ce n'est pas une question de “taper”, je n'aime pas “taper” sur mon ministère, ça me rend malheureuse... Je n'essaie pas de dire $\mathrm{X}$ est plus beau qu'Y. Ça ne m'intéresse pas... je suis une grande joueuse de bridge, et en travaillant, je fonctionne pareil. J'essaye d'étudier le maximum de paramètres et d'hypothèses possibles, avant de décider la carte que je vais abattre. Mais bon ceci dit, c'est vrai que toujours, avant de dire les militaires ont raison ou le Quai devrait changer sa position, je vais d'abord voir le Quai en disant “on fait fausse route”. »

(Entretien avec l'ancienne directrice financière de la cellule d'urgence et secrétaire générale de la MIESE, Paris, 25 octobre 2009)

Au-delà de leurs liens avec l'univers associatif et humanitaire, la principale ressource de ces acteurs est éminemment politique : elle tient à leur pleine insertion au sein des univers socialistes de la gauche mitterrandienne. Si le directeur opérationnel connaît Bernard Kouchner dès la fin des années 1970, il travaillera directement avec lui entre 1988 et 1993, 1998 et 2002, tandis que la directrice financière bénéficie déjà d'un contact privilégié avec le couple Mitterrand dès la fin des années 1960.

«Q-A propos de Mme Mitterrand... vous m'aviez dit que vous l'aviez rencontrée dans les années $80 .$.

«Avant 1980.

"Q-Donc vous la connaissiez avant que son mari n'accède à la plus baute responsabilité?

«Oui... disons que euh... je rencontre les Mitterrand sur un sujet culturel qui n'a rien à voir avec tout ça... en 1967 ou 1968 et puis je 
ne les avais pas revus après... mais je les avais rencontrés chez eux, donc c'était quand même assez privé. Et puis j'ai fait la campagne de François Mitterrand, mais je n'étais pas membre du PS...

«Q- Vous êtes militante socialiste aujourd'bui?

«Oui, à l'époque je ne l'étais pas... je suis une femme de gauche, ça c'est vrai...

"Q-Que faisiez-vous pendant cette campagne?

"Je m'occupais des Français de l'étranger et j'ai participé à la création du BFE [Bureau des Français de l'étranger]... »

(Entretien avec l'ancienne directrice financière de la cellule d'urgence et secrétaire générale de la MIESE, Paris, 25 octobre 2009)

C'est en s'intéressant au parcours de Jean-Maurice Ripert et Roger Fauroux que l'on peut saisir l'importance des réseaux qui permettent à ces acteurs de perdurer dans un environnement institutionnel plutôt hostile. Précisons que la création de la MIESE a été impulsée par Jean-Maurice Ripert, alors conseiller diplomatique de Matignon et actuel ambassadeur de France en Russie. Il nous a d'ailleurs été rapporté le fait qu'il aurait été l'un des principaux artisans de la création du SAH quelques années plus tôt. Sous le gouvernement de Michel Rocard, Jean-Maurice Ripert, alors déjà conseiller diplomatique à Matignon, est chargé, selon le directeur opérationnel, de "défendre le SAH de Bernard Kouchner contre le Quai d'Orsay ». Ce n'est semble-t-il pas tout à fait un hasard si, retrouvant son poste de conseiller diplomatique en 1997, Jean-Maurice Ripert contribue à renommer le directeur opérationnel de la cellule d'urgence, puis la secrétaire générale et le président de la MIESE. Il est issu de la promotion «Voltaire » (1980) de l'École nationale d'administration (ENA) avec, entre autres ténors du Parti socialiste, Ségolène Royal, Michel Sapin et François Hollande. Ancien président-directeur général de Saint-Gobain (1961-1986), directeur de l'ENA (1986-1988), ministre de l'Industrie, du Commerce extérieur et de l'Aménagement du territoire du gouvernement Michel Rocard (1988-1991) au même moment que Bernard Kouchner au SAH, le parcours de Roger Fauroux, témoignant d'une connaissance du fonctionnement des milieux publics et privés, est sans doute l'une des clés de sa nomination à la tête de la MIESE. Il est à la même époque président de la Fondation Saint-Simon, créée en 1981, dont les objectifs sont multiples : dresser un pont entre l'univers des entreprises et de la politique au moment de la « grande peur » de l'arrivée de la gauche au pouvoir, marquer l'indissociabilité de la démocratie et de l'économie de marché ${ }^{42}$. De fait, ces réseaux touchent aussi le monde de l'industrie, la fondation comptant un certain nombre de chefs d'entreprises, de journalistes et un acteur humanitaire alors très en

42. Membres du conseil d'administration : Jean-Claude Casanova, Roger Fauroux, Alain Minc, Jean Peyrelevade, Pierre Rosanvallon. Contributeurs : Suez, Publicis, la Sema, le Crédit local de France, la banque Wormser, Saint-Gobain, BSN Gervais-Danone, MK2 Productions, Cap Gemini Sogeti, Laurent V., "Les architectes du social libéralisme ", Le Monde diplomatique, septembre 1998, pp. 26-27. 
vue, Bernard Kouchner. Autrement dit, les urgentistes du MAE sont reliés depuis plusieurs décennies à des réseaux d'affinités électives touchant le plus haut niveau politique.

\section{Les professionnels de gestion des crises internationales}

Des officiers des forces spéciales, ni tout à fait militaires, ni espions, ni industriels qui forment une nébuleuse fonctionnant discrètement par cooptation de réseaux et disposant de capacités de reconversion certaines. Des officiers généraux héritiers de l'armée d'Afrique aux parcours sans tâche qui refusent de se contenter de l'immobilisme de la non-guerre nucléaire, qui trustent les positions d'autorité au sein d'une armée réformée et deviennent les cadres du maintien de la paix. Des urgentistes du MAE, plus «opérationnels » que « diplomates », militants socialistes tournés vers l'univers des ONG, qui se protègent mutuellement en s'aménageant, dans la difficulté, un « espace de liberté » au sein de leur institution. Nous souhaitons ouvrir ici les perspectives de recherche en passant de l'évaluation des apports respectifs des différents groupes professionnels à l'institutionnalisation des activités civilo-militaires à l'analyse systématique des effets de la routinisation de leurs interactions sur la transformation de la politique étrangère. Nous souhaitons affermir l'hypothèse intersectorielle : l'intelligibilité des processus liés à la transformation de la politique étrangère passe d'abord par l'observation concrète de l'évolution des relations entre acteurs et entre secteurs. Voilà pourquoi nous soutenons une démarche inductive qui ne part pas uniquement du constat de la transformation de l'environnement international mais de l'évolution des positions et des formes d'interactions entre groupes, ici militaires, diplomates et industriels.

\section{Caractéristiques trans-sectorielles}

Si l'on croise les trois groupes disséqués jusqu'à présent en se plaçant au niveau des trajectoires individuelles et des ressources collectives, on obtient certaines caractéristiques trans-sectorielles qui pourraient permettre de dessiner les contours d'un groupe de gestionnaires de crises internationales en voie d'autonomisation. Un certain nombre de points communs liés à la socialisation primaire ont été souligné à propos des deux premiers groupes d'acteurs en compétition pour le leadership des activités civilo-militaires : image du père, tradition familiale au service de l'État, prédisposition à la mobilité internationale, connaissance personnelle de l'Afrique ${ }^{43}$.

43. À propos du secteur humanitaire, on relève aussi la figure marquante du père, « modèle » ou « héros ». Siméant J., « Entrer, rester en humanitaire. Des fondateurs de Médecins sans frontières aux membres actuels des ONG médicales françaises ", Revue française de science politique, 51/1-2, 2001, pp. 47-72. 
« Et donc ma carrière militaire elle a pour origine... bon d'abord l'admiration que j'avais pour mon père, mon père était un personnage, à mon avis, hors série, et donc quand on a la chance d'avoir un père comme ça, bah il vous marque, forcément... et de manière très humaine. Je me souviens que je devais avoir treize ou quatorze ans à Djibouti, il y avait des mouvements de frontière avec la Somalie, et je me souviens de mise en alerte de nuit des éléments blindés, fantassins et artillerie, et mon père m'embarquait toujours dans sa jeep et je partais avec lui. Je n'avais pas de plus grand plaisir que de mettre une tenue camouflée, de me glisser dans le fond de cette jeep et d'aller assister dans un petit coin au briefing, aux ordres donnés, etc., donc c'est vrai, j'ai contracté le virus très jeune, en Afrique. » (Entretien avec un ancien colonel « affaires civiles » du COS, Paris, 13 octobre 2009 )

La notion d'engagement est souvent évoquée en des termes prosaïques : il s'agit du goût de l'aventure, non pas au sens de la mise en danger ou de l'exposition physique de soi, mais plutôt au sens de l'envie de "voir du pays ", de «bouger » et de «trouver un cadre ». Les arguments plus «classiques » (le patriotisme, la notion de service de l'État, la tradition familiale...) n'étant mobilisés qu'en dernier recours, généralement suite à nos relances.

\begin{abstract}
«Moi je suis rentré dans l'armée tout simplement parce que conformément à mon tempérament, c'est l'aventure, le risque, le sport... voilà... il ne m'est jamais venu à l'esprit de faire un métier intellectuel, de faire un doctorat, j'aime autant vous dire que ça n'était pas dans mon état d'esprit. Donc bon, voilà mon histoire. Vous savez ma démarche, moi au départ militaire... ce n'est pas militaire... c'est l'aventurier... le sport... j'étais très sportif alors... »

(Entretien avec un colonel de l'armée de terre, Paris, 3 avril 2008)
\end{abstract}

Ces acteurs se présentent en tant qu' « opérationnels » par opposition aux technocrates en liant leurs parcours à la «prise de risque », à la fois entendue comme éthique individuelle et spécialité professionnelle. Le risque n’est pas seulement lié à la nature même des opérations menées et aux contextes dans lesquels elles se déroulent, sa « gestion » est un élément de distinction sociale ${ }^{44}$. L'assimilation réciproque entre ces trois groupes d'acteurs se fait sur les «terrains » ou «théâtres » : pour ces « opérationnels », il s'agit d'un espace de socialisation mais aussi d'identification par constitution d'une mémoire commune à partir d'expériences partagées dans des contextes parti-

44. «Sur quelles ententes les volontaires de l'humanitaire fondaient-ils leur travail commun ? Ils étaient plus différents que semblables et s'ils poursuivaient un temps ensemble leur chemin d'aventure c'était avant tout pour pouvoir profiter de cette organisation rationnelle du frisson. [...] Ainsi malgré nos ironies et nos doutes nous avions créé une aristocratie du risque... », Kouchner B., Le Malheur des antres, Paris, Odile Jacob, 1992, p. 327. 
culièrement informels et tendus. Si ces acteurs en arrivent à développer des méthodes de contournement des pesanteurs bureaucratiques, ils forgent une sorte d'éthique du «système $\mathrm{D}$ » dont l'imagination et la débrouillardise sont les piliers. On note un réel enthousiasme à l'idée d'improviser dans l'urgence en marge des procédures:

"Vous vous permettez tout parce que vous arrivez avec des méthodes associatives... des méthodes qui décoiffent... tout ça appris dans l'univers associatif, et comme on venait des ONG on avait pas mal d'idées par rapport aux administrations sclérosées... parce qu'avec tous les ministères vous finissez broyés autrement... je me souviens, je téléphone à Renault... est-ce que vous pouvez me passer une fourgonnette pour le tremblement de terre [en Arménie, décembre 1988] ? Ah bon on peut être utile ? D'accord, on vous envoie ça... Allo Peugeot, ici c'est le secrétariat d'État à l'Action humanitaire, vous pouvez nous dépanner ? La même chez Manpower... j’ai besoin d'une assistante... et le directeur de la communication qui me dit: "vous êtes l'État et vous me demandez ça à moi ?”. Je lui dis oui et alors ? Le mec éclate de rire... j’ai gardé les camionnettes pendant six mois et l'assistante pendant un an et demi...»

(Entretien avec l'ancien directeur opérationnel de la cellule d'urgence, Paris, 15 décembre 2009)

Manifestant leur capacité à évoluer dans des contextes déroutants, ce sont ces mêmes «terrains » ou «théâtres » qui contribuent, à travers la constitution d'une mémoire partagée, à forger une éthique antibureaucratique. Un trait commun est frappant : leur pire adversaire, dans l'exécution de leurs tâches quotidiennes, est le supérieur ou le bureaucrate. Il s'agit de changer les choses de l'intérieur ; la personne à convaincre n'étant généralement pas une entité abstraite et éloignée (le cabinet du ministre, la présidence de la République, l'OTAN, l'opinion publique...) mais son propre " patron », chacun à son échelle, dans son organisation de travail. Au-delà d'un effet générationnel crucial - ces acteurs appartiennent à la cohorte des «papy boomers »c'est sans doute la position de certains acteurs-clés, à l'intérieur de leur secteur respectif, qui a pu faciliter les échanges intersectoriels. En premier lieu, ces acteurs subissent et portent depuis les années 1980, au sein de leur institution, des transformations organisationnelles proches en termes de technicisation et de bureaucratisation. Ensuite, la position de marginal-sécant de certains d'entre eux facilite les transactions collusives entre secteurs, et donc l'établissement de rapports de légitimation et de domination davantage horizontaux que verticaux. Par ailleurs, relativement rare avant les années 1980, la mobilité intersectorielle sera très forte à partir de la fin des années 1990, à l'image de la reconversion déjà évoquée d'un certain nombre d'officiers civilo-militaires et 
de fonctionnaires du MAE au sein d'OI, d'ONG et d'entreprises. Elle dénote une forte fongibilité des capitaux et une capacité certaine de cooptation en réseaux.

\section{Espace, groupe, champ}

Si l'homologie des trajectoires professionnelles des acteurs de la gestation des activités civilo-militaires françaises en ex-Yougoslavie est selon nous un indice crucial de la recomposition sectorielle de la politique étrangère depuis la fin de la guerre froide, encore nous faut-il caractériser le phénomène observé. Que forment ensemble les acteurs de gestion de crises internationales ? S'agit-il d'un espace interministériel, d'un groupe professionnel ou même d'un champ social en voie de structuration au sein de la politique étrangère ? Évoquer l'émergence d'une espace interministériel reviendrait en quelque sorte à faire de la coopération civilo-militaire hier et de «l'approche globale » aujourd'hui une simple technologie administrative de partage d'informations. Cette intuition n'est pas absurde, à ceci près, nous l'avons vu, que les gestionnaires de crises partagent plus que des informations, des normes et des techniques. Peut-on alors parler d'une « colonisation» entre segments institutionnels dont l'intégration serait la réponse bureaucratique aux effets de la convergence des programmes de sécurité, de gouvernance et de développement sur les zones d'intervention 45 ? Là encore la vitalité des rivalités interministérielles autour d'enjeux de culture administrative et de périmètres d'action invite à la prudence tandis que pour nous, l'intégration interministérielle devance la convergence des programmes internationaux et non pas l'inverse. Avons-nous alors affaire à une « agrégation 46 » de groupes professionnels qui serait notamment favorisée depuis une trentaine d'années par la mise en œuvre des réformes du New public Management 47 ? Cette piste nous semble intéressante dans la mesure où les dynamiques qui affectent la bureaucratisation de la sécurité internationale pourraient être comparées à celles qui touchent la sécurité sanitaire. Les travaux sur la transformation du secteur hospitalier offrent ainsi un exemple d'investigation utile à plusieurs niveaux : constatant eux aussi un élargissement de la notion de sécurité du fait de la multiplication des «crises » sanitaires au cours des années 1990, ils tentent de cerner les contours d'un champ à partir des recompositions sectorielles entre différents groupes professionnels 48 et des effets de l'émergence du modèle organisationnel des

45. Chandler D., "The security-development nexus and the rise of 'anti-foreign policy'”, Journal of International Relations and Development, 10/4, 2007, pp. 362-386 ; Neal D., Capability Development in Support of Comprehensive Approaches. Transforming International CivilMilitary Interactions, Center for Technology and National Security Policy, Institute for National Strategic Studies, National Defense University, Décembre 2011.

46. Bucher R., Strauss A., "Professions in Process", American Journal of Sociology, 66/4, 1961, pp. 325-334.

47. Bezes P., Réinventer l'État. Les réformes de l'administration française (1962-2008), Paris, PUF, 2009.

48. Pierru F., «Le mandarin, le gestionnaire et le consultant. Le tournant néolibéral de la politique hospitalière ", Actes de la recherche en sciences sociales, 194, 2012, pp. 32-51. 
agences de l'État ${ }^{49}$. On ne peut que déplorer ici l'extraterritorialité des objets «stratégiques » au regard des sciences sociales tant les pistes liées au Policy Transfer et à l'évolution des relations entre pouvoir politique et technocratique nous semblent stimulantes 50 .

Pour ce qui est de l'hypothèse d'un champ en voie de structuration, force est de constater que l'émergence progressive d'un « espace » ou d'un « groupe » de gestion de crises internationales n'a pas entraîné la fin de l'autonomie des secteurs traditionnels de la politique étrangère qui «fonctionnent » encore sur des logiques différentes voire contradictoires. La période postbipolaire a vu le remplacement du terme de « défense » (lié aux «menaces » externes) par celui de " sécurité » (liée aux « risques » internes). Le champ de la « sécurité », « gérant » désormais des « risques » et non plus des « menaces », est exposé aux «crises » et non plus simplement aux « guerres»51. Si l'utilisation de la dichotomie menaces/risques reste largement métaphorique dans la plupart des arènes politico-militaires, les « risques » constituent, en tout cas du point de vue de la planification stratégique, des « menaces » jugées impossibles à endiguer par la force militaire en tant que telle. Il s'ensuit évidement l'inévitable exercice taxinomique qui décline les sécurités : humaine, sociale, environnementale, alimentaire, sanitaire... Cette mise en perspective nous invite à poser la question du caractère conjoncturel ou structurel des transformations d'un champ. Ainsi, si certaines analyses, notamment à travers une approche $\mathrm{du}$ « changement » par les mobilisations multisectorielles, plaideraient pour une désectorisation conjoncturelle depuis la crise post-bipolaire, d'autres mettent en avant des processus de civilianisation et d'intégration de nouveaux acteurs depuis la fin de la Seconde Guerre mondiale et l'avènement des armes nucléaires.

L'hypothèse de la structuration d'un champ de gestion de crises internationales nous invite à nous saisir des enjeux liés à la division interministérielle du travail international. La genèse des activités civilo-militaires en France ne nous renseigne pas uniquement sur la teneur des rivalités interministérielles et les mobilisations qui les accompagnent. Elle montre clairement que le caractère volatil du leadership politique en matière de gestion de crises internationales est lié à la vitalité du consensus non décisionnel qui entoure cette divi-

49. Benamouzig D., Besançon J., « Administrer un monde incertain : les nouvelles bureaucraties techniques. Le cas des agences sanitaires en France », Sociologie du travail, 47, 2005, pp. 301322.

50. Les administrations militaires et diplomatiques constituent souvent un « angle mort » des politiques publiques. À de notables exceptions en France, le changement institutionnel y est étudié comme un processus décisionnel "top-down », une sorte de " chasse gardée élyséenne ». Genieys W., L'Élite des politiques de l'État, Paris, Presses de Sciences Po, Paris, 2008 ; Irondelle B., La Réforme des armées en France. Sociologie de la décision, Paris, Presses de Sciences Po, 2011 ; Hamelin F., «Le combattant et le technocrate. La formation des officiers à l'aune du modèle des élites civiles », Revue française de science politique, 53/3, 2003, pp. 435-436; Meimon J., "Culte du terrain à la rue Monsieur. Les fonctionnaires de la France d'outre-mer et de la coopération », Afrique contemporaine, 236, 2010, pp. 53-65. 
sion interministérielle du travail. Le consensus non décisionnel n'est pas réductible au fait de " choisir de ne pas choisir », en l'occurrence entre les ministères : envisagé de façon « positive », il permet de comprendre comment l'équilibre interministériel, certes instable, évolue de façon incrémentale 52. Nous entendons par consensus non pas un «compromis» ni un accord sur le sens ou les valeurs, mais plutôt une « règle pragmatique 53 » dont il est « coûteux de s'écarter trop ostensiblement ${ }^{4}$ ». L'intentionnalité des acteurs dans son émergence et sa reconduction est limitée même s'il se trouve être l'objet d'incessantes tentatives de débordements dont l'institutionnalisation de la CIMIC entre 1992 et 2005 ou la formalisation de l'approche globale depuis 2010 constituent des exemples concrets. Au-delà des usages et des règles constitutionnelles, la localisation du pilotage de gestion des crises internationales en France dépend essentiellement de deux facteurs : l'inscription de la zone d'intervention dans les routines organisationnelles des ministères 55 et l'évolution du niveau d'insécurité et donc de la nature, civile ou militaire, de la majorité des effectifs et des moyens mobilisés 56 .

Les « récits de vie » mobilisés dans cet article sont issus d'une cinquantaine d'entretiens appuyés par le dépouillement de nombreuses archives. Ils ne doivent cependant pas nous amener à surévaluer la rationalité et l'intentionnalité des acteurs dans la reconstitution de leurs trajectoires et de leurs mobilisations au cour des réformes ${ }^{57}$. L'objectif demeure la réincarnation de l'analyse du changement institutionnel au sein des appareils bureaucratiques de la politique étrangère et de défense. Ces « récits » n’ont d'intérêt heuristique que rapportés au réductionnisme opéré en amont. L'exemple de la genèse des activités civilo-militaires en ex-Yougoslavie offre un angle empirique qui permet de tenter une explication alternative du changement. L'originalité tient avant tout à la démarche inductive qui tempère le fonctionnalisme de la thèse de l'adaptation des organisations et des acteurs aux transformations de l'environnement international depuis la fin de la guerre froide. D’un côté, la fin de la

51. Autant d'évolutions sémantiques, palpables dès 1994 et manifestes dès 2008 ( $c f$. les titres mêmes des Livres Blancs de la Défense et la sécurité nationale de 2008 et 2013).

52. Bachrach P., Baratz M., "Decision and Nondecision: an analytical framework", The American Political Science Review, 57/3, 1963, pp. 632-642.

53. Bailey F., Les Règles du jeu politique, Paris, PUF, 1971.

54. Dobry M., « Le jeu du consensus », Pouvoirs, 38, 1986, pp. 47-66.

55. La division du travail par zone géographique semble se répartir comme suit : les interventions en Afrique au Mali et en Centrafrique sont du ressort de la Défense (accords bilatéraux hérités de l'ère coloniale, principale force militaire occidentale prépositionnée, connaissance de la zone...) et les (non-)interventions au Moyen-Orient, comme en Syrie ou en Iran, sont du ressort du MAE (organisation en bureau géographique, représentations dans les enceintes multilatérales, puissance des réseaux diplomatiques locaux...).

56. La création et la montée en puissance de la cellule AFPAK sous leadership du MAE à partir de 2009 au moment du resserrement du dispositif militaire autour de la Task Force La Fayette en Afghanistan et parallèlement, le déroulement des récentes interventions au Mali et en Centrafrique entièrement gérées par la Défense, semblent confirmer la vitalité de ce consensus non décisionnel.

57. Simon H., "Human Nature in Politics: The Dialogue of Psychology with Political Science”, American Political Science Review, 79/2, 1985, pp. 293-304. 
régulation de la conflictualité internationale entre les deux superpuissances agit comme contrainte puisque la dissuasion ne suffit plus à garantir la sécurité, que la possibilité d'une guerre interétatique majeure est devenue obsolète et que les ennemis sont désormais non-étatiques. De l'autre, les autorités ont répondu à cette mutation de l'environnement international par la refonte de leurs capacités de projection via la mise en œuvre des réformes de la défense entre 1992 et 1996. Il nous paraît tout à fait primordial de préciser ici que nous ne sombrons pas dans l'excès inverse de la théorie de l'adaptation qui consisterait en une sorte de « théorie du complot ». Ce n'est pas parce que nous réfutons le déterminisme de la thèse de l'ajustement homéostatique des organisations militaires aux transformations de l'environnement que nous considérons au contraire que l'institutionnalisation de la CIMIC est le résultat des qualités d'anticipation tactique ou de clairvoyance stratégique de quelques-uns, précisément les futurs officiers CIMIC. Cette démarche nous invite plutôt à considérer la place du " hasard », ou de façon moins relativiste, le caractère hautement indéterminé des processus d'institutionnalisation. Il s'agit donc de saisir la genèse des activités civilo-militaires, comme une trajectoire non prédéterminée - et surtout non nécessaire - entre différents carrefours historiques possibles et « ballotée » entre des appropriations très hétérogènes, afin de rompre radicalement avec le déterminisme évolutionniste et fonctionnaliste de la thèse de l'adaptation. 